\title{
Correlation between thyroid function, testosterone levels, and depressive symptoms in females with spinal cord injury
}

\author{
Benjamin Dirlikov ${ }^{1} \cdot$ Sarah Lavoie ${ }^{1} \cdot$ Kazuko Shem $^{1,2}$ \\ Received: 5 December 2018 / Revised: 3 May 2019 / Accepted: 5 May 2019 \\ (c) The Author(s), under exclusive licence to International Spinal Cord Society 2019
}

\begin{abstract}
Study Design Prospective case series.

Objective Investigate the association of testosterone and thyroid-stimulating hormone (TSH) levels with depressive symptoms in women after spinal cord injury (SCI).

Setting Community SCI clinic.

Methods Twenty-seven participants were enrolled in this study. Total testosterone (Total T) and TSH levels as well as the Center for Epidemiological Studies Depression Scale (CES-D) survey and monthly sexual activity were obtained from only 20 participants. Pearson's correlations were used to assess the relationship between age, time from injury, Total T level, TSH level, and CES-D total score. Follow-up analyses investigating the role of monthly sexual activity was also explored.

Results Participants' average age and time from injury was $44.4 \pm 12.7$ years old and $11.7 \pm 8.89$ years, respectively. Low Total T was observed in four participants and one of those participant's presented with low TSH as well. Nine women were classified as "at risk for clinical depression" on the CES-D (total score $>15$ ). Pearson's correlations revealed a significant association between time from injury and TSH $(r=.536, p=.015)$, as well as CES-D total score $(r=-.547, p=.013)$. Total T was associated with CES-D total score $(\rho=-.541, p=.02)$.

Conclusions This study provides preliminary results on abnormal hormone levels and depressive symptoms in women after SCI. Twenty percent of this sample presented with low Total T, which was associated with increased depressive symptoms after accounting for time from injury. Further research is needed to investigate the impact of SCI on hormone function and mental health in women post SCI.
\end{abstract}

Sponsorship Sally Rynne National Association of Women's Health Quality Award 2002.

\section{Introduction}

In 2018, approximately 288,000 individuals were living with a spinal cord injury (SCI) [1]. An estimated 78\% of new SCI cases are male [1], resulting in research skewed towards male-specific issues. The paucity of research relevant for women with SCI is also due to the neglect of

These authors contributed equally: Benjamin Dirlikov, Sarah Lavoie

Kazuko Shem

Kazuko.Shem@hhs.sccgov.org

1 Rehabilitation Research Center, Santa Clara Valley Medical Center, San Jose, CA, USA

2 Department of Physical Medicine and Rehabilitation, Santa Clara Valley Medical Center, San Jose, CA, USA researchers to stratify results by gender [2, 3]. For these reasons unique concerns for women with $\mathrm{SCI}$ have not been adequately identified or addressed. In spite of considerable efforts to improve this disparity over the past 20 years, significant work remains [2, 4].

Significant changes in testosterone levels [5] have been noted in males with SCI [6-9]. Compared to able-bodied normative data, males with SCI show a higher prevalence of low serum total testosterone (Total $\mathrm{T}$ ) and greater agerelated declines. SCI may also elicit "accelerated aging" in males through the development of metabolic dysfunction and subsequent testosterone deficiency [5]. A retrospective study by Bauman et al. [10] found that $46 \%$ of males with SCI $(n=243)$ presented with low testosterone; low testosterone has also been identified in veterans with SCI (43\%; $n=60$ ) [11]. Predictors of low testosterone in men with chronic SCI include high body mass index, more extensive injuries [5], poor leisure time, physical activity, and low 
sexual desire [12]. A review of the literature found only one study investigating hormonal changes in women after SCI, of which no significant correlation was found between abnormal hormonal status and sexual dysfunction [13]. In this sample, 6 out of $39(\sim 15 \%)$ showed abnormal hormone levels; 2 out of $39(\sim 5 \%)$ presented with low Total T.

Thyroid-stimulating hormone (TSH) is known to affect the metabolism of testosterone and all androgens through mediating sex hormone-binding globulin levels, which alters levels of free and bound testosterone [14]. In ablebodied females, significant correlations between hypothyroidism concomitant with decreased androgen metabolism and depression have been reported [15, 16], and can be ameliorated by administering serum thyroxine (T4) with or without triiodothyronine (T3) [15-17]. Decreased serum testosterone levels have been associated with diminished sexuality [18] and increased depression in able-bodied women [15-17]. Research has also found evidence to support the neuroprotective role of testosterone in neurodegenerative disorders including depression; testosterone's neuroprotective effects may be mediated both indirectly (via the conversion of testosterone to estrogen) and directly (acting through the androgen pathway) [19, 20].

Although thyroid function has been shown to be lower in some individuals with SCI, most of these studies were conducted among males [8, 9, 21, 22]. Previous research has found $\mathrm{T} 3$ levels to be significantly decreased compared to TSH or T4 levels in males with chronic SCI, which suggests that the males were clinically euthyroid $[8,21]$. Only one study of 14 females with SCI examined thyroid function, of which normal thyroid function was reported [22]. As with testosterone, decreased thyroid function has been correlated with depression in the able-bodied population $[15,23]$.

Research exploring the relationship between testosterone levels and depression in women with SCI is limited. In the general population research has established that clinical endocrine disorders, including both the hypo- and hyperfunction of endocrine glands, often result in psychiatric symptoms, with depression being the predominant clinical presentation [24]. Able-bodied females experience depression at higher rates than able-bodied males, suggesting that hormones may play a role in the etiology of the disease [25]. Furthermore, both low and high levels of testosterone in able-bodied women have been linked to depression [25]. In women without SCI, levels of total and free testosterone decline with age as women enter pre-menopause and may contribute to decreased mood [26].

Depression prevalence is significantly higher among persons with a SCI as compared to the able-bodied population [27]. A meta-analysis by Williams and Murray [27] reviewed 19 studies; depression prevalence was estimated to affect $18.7 \%$ to $26.3 \%$ of persons with SCI compared with an estimated $16 \%$ lifetime depression prevalence among the general able-bodied population [28]. Factors contributing to depression risk in individuals with SCI include: female gender, socioeconomic status [29], younger age [30], social isolation [31], alcohol and substance abuse [32], and pain [33]. Greater time since injury [34], resilience [35], self-efficacy [36], and social support [37] are associated with reducing depression risk. Previous research has also linked hypothyroidism $[15,16]$ and low testosterone levels [15-17] to an increased risk for depression among the able-bodied population. Given the high estimates of depression following SCI and the limited number of studies assessing the influence of thyroid and testosterone hormones on depression risk among women post SCI, this study sought to address this gap in knowledge.

The objective of this study was to measure thyroid function and serum testosterone levels, and to correlate these results with indicators of depression in females with SCI. We hypothesize that depression will be correlated with hormone levels, such that abnormal hormonal status will be associated with increased depressive symptoms. Furthermore, we will explore the association between sexual function and hormone levels, as well as depressive symptomology.

\section{Methodology}

This study was approved by the local Institutional Review Board of the study site, which is a county hospital in a suburb of California. Participants were recruited if they were females with SCI of any etiology and any ethnicity. The inclusion criteria were: (1) at least 1 year post SCI; (2) neurologically and medically stable; and (3) at least 18 years of age. The exclusion criteria were: (1) significant medical problems, such as pneumonia, kidney failure, febrile urinary tract infection, other febrile diseases; (2) current or prior medical history of head injury, thyroid dysfunction, and known endocrine disorders, (3) pregnancy, and (4) inability, due to language difficulties, to understand the informed consent and questionnaires. Prior to the initiation of any study procedure, each participant read and signed an informed consent. Twenty-seven participants were enrolled but only 22 had complete hormone and questionnaire data. Of the 22 patients, 2 participants had outlier serum Total $\mathrm{T}$ levels $(0$ and $89 \mathrm{ng} / \mathrm{dl})$; therefore, the remaining 20 participants were used in this analysis. Participant demographics are listed in Table 1 and concomitant medications are listed in Table 2. Each participant was asked to fill out a survey that included demographic questions, a question on monthly sexual activity, and the Center for Epidemiological Studies Depression Scale (CES-D) (described below) [38]. The survey question "How often do you have sexual activity per 
Table 1 Demographics

\begin{tabular}{lll}
\hline Demographics & Mean & Std \\
\hline Age (years) & 44.4 & 12.7 \\
Years from injury & 11.7 & 8.9 \\
Neurologic status & Count & \\
Complete SCI & 6 & \\
Incomplete SCI & 14 & \\
Level of injury & & \\
Cervical & 13 & \\
Thoracic & 6 & \\
Lumbar & 1 & \\
Etiology of injury & & \\
MVA & 14 & \\
GSW & 1 & \\
Non-traumatic & 4 & \\
Water sports & 1 & \\
Monthly sexual activity & & \\
Yes & 9 & \\
No & 11 & \\
Std & &
\end{tabular}

Std standard deviation, SCI spinal cord injury, MVA motor vehicle accident, $G S W$ gunshot wound

month?" was dichotomized to a yes/no response since over half the sample (11/20) women responded 0. Monthly sexual activity ranged from 0 to 29 times per month, with all but one woman responding $<5$ times per month (Table 1).

A blood sample was drawn to measure thyroid function, which included TSH and serum Total T levels. Due to diurnal variations in testosterone levels, blood samples were drawn between 10:00 a.m. to 12:00 p.m. for consistency [39]. Testosterone levels reach their peak during the late follicular and early luteal phase [18]; therefore, testosterone levels were drawn within the first 5 days of the menstrual cycle, if a participant was not post-menopausal.

\section{Center for Epidemiological Studies Depression Scale}

The CES-D is a 20-item scale asking respondents to indicate the number of days during the past week they have experienced symptoms like feeling fearful, happy, or lonely. Four subscale scores-depressive affect, well-being, somatic symptoms, and interpersonal affect-as well as a total score were obtained. Total CES-D scores range from 0 to 60 , with a higher score indicating more depressive symptomatology. A score ranging from 0 to 7 is defined as having "clinically irrelevant symptoms," 8 to 15 as "subclinical depressive symptoms," and scores of 16 or greater are defined as having "clinically relevant levels of
Table 2 Concomitant Medications

\begin{tabular}{|c|c|c|}
\hline Indication & Medication & Count \\
\hline Allergy nasal symptoms & Fluticasone & 1 \\
\hline \multirow[t]{3}{*}{ Analgesic/antipyretic } & Acetaminophen & 2 \\
\hline & Aspirin & 2 \\
\hline & Ibuprofen & 3 \\
\hline \multirow[t]{3}{*}{ Antacid } & Cimetidine & 1 \\
\hline & Calcium carbonate & 1 \\
\hline & Omeprazole & 1 \\
\hline \multirow[t]{3}{*}{ Antibiotic } & Cephalexin & 2 \\
\hline & Neosporin & 1 \\
\hline & Nitrofurantoin & 1 \\
\hline $\begin{array}{l}\text { Antihistamine for sinus } \\
\text { allergy }\end{array}$ & Cetirizine & 1 \\
\hline \multirow[t]{2}{*}{ Benzodiazepine } & Clonazepam & 1 \\
\hline & Lorazepam & 1 \\
\hline Birth control & $\begin{array}{l}\text { Norethindrone/Ethinyl } \\
\text { Estradiol }\end{array}$ & 1 \\
\hline Bloating & Simethicone & 1 \\
\hline \multirow[t]{3}{*}{ Bowel program } & Senna glycoside & 2 \\
\hline & Theravac & 1 \\
\hline & Docusate sodium & 2 \\
\hline \multirow[t]{5}{*}{ Dietary supplement } & Hexapio Ginsena & 1 \\
\hline & Multivitamin & 2 \\
\hline & Vitamin B6 & 1 \\
\hline & Vitamin C & 1 \\
\hline & Vitamin E-PRN & 1 \\
\hline Low blood calcium & Calcium Carbonate & 1 \\
\hline Heterotopic ossification & Etidronate & 1 \\
\hline $\begin{array}{l}\text { Hypertension/urinary } \\
\text { retention }\end{array}$ & Doxazosin & 1 \\
\hline Insomnia & Zolpidem & 1 \\
\hline \multirow[t]{2}{*}{ Muscle spasms/spasticity } & Baclofen & 7 \\
\hline & Tizanidine & 1 \\
\hline \multirow[t]{2}{*}{ Opioids for pain } & Morphine Sulfate & 2 \\
\hline & Oxycodone & 1 \\
\hline Neurogenic bladder & Oxybutynin & 11 \\
\hline Neuropathic pain & Gabapentin & 4 \\
\hline Osteoporosis & Alendronic acid & 1 \\
\hline \multirow[t]{3}{*}{ Post-menopausal symptoms } & Estradiol-Norethindrone & 1 \\
\hline & Estrogen & 3 \\
\hline & Progesterone & 1 \\
\hline \multirow[t]{3}{*}{ Depression/Anxiety } & Fluoxetine & 1 \\
\hline & Sertraline & 1 \\
\hline & Duloxetine & 1 \\
\hline Sexual dysfunction & Sildenafil & 1 \\
\hline None listed & & 7 \\
\hline
\end{tabular}

Summary of concomitant medications in sample. Count represents the number of participants taking the medication 
depressive symptoms." The CES-D has been widely used within community populations, including older adults, to assess depressive affect and has been shown to correlate significantly with clinical depression ratings [38, 40-43].

\section{Statistical analyses}

Due to well-established changes in depression ratings and hormone levels following SCI, Pearson's correlations were used to assess the relationship between time from injury (years) and age with TSH, Total T, and CES-D total score. Significant associations between time from injury and CES-D total scores, as well as TSH were found, and, therefore, partial correlations controlling for time from injury were used when correlating CES-D total scores to serum Total T and TSH. An exploratory analysis was conducted to investigate whether individuals who endorsed having monthly sexual activity had (1) differences in hormone levels (TSH and Total T; Mann-Whitney $U$ test) and (2) lower rates of clinically relevant levels of depressive symptoms (CES-D total $>15$; Fisher's exact test) compared to individuals who did not have monthly sexual activity. All statistical analyses were run using IBM's SPSS statistical software (version 24) with a significance level set at $p$ less than or equal to .05 .

\section{Results}

\section{Demographics}

A total of 20 females with SCI were included in this analysis (Table 1). Average age was $44.4(\mathrm{SD}=12.7)$ years old and average time since injury was $11.7(\mathrm{SD}=8.9)$ years. Six individuals had complete SCI and 14 had incomplete SCI. Thirteen individuals had a cervical injury, six had thoracic injuries, and one had a lumbar injury. Etiologies of SCI were: motor vehicle accident $(n=14)$, violence/gunshot wound $(n=1)$, non-traumatic $(n=4)$, and water sports $(n=1)$. Nine participants were post-menopausal, four of which had an oophorectomy.

\section{Hormone assay}

Average TSH level was $1.34 \mathrm{mcIU} / \mathrm{ml}(\mathrm{SD}=0.76)$ (normal range: $0.20-5.39 \mathrm{mcIU} / \mathrm{ml}$ ) in this sample. Only one participant presented with a TSH level below the normal range $(0.12 \mathrm{mcIU} / \mathrm{ml})$. Due to heterogeneity in the sample, different low Total $\mathrm{T}$ thresholds were used for participants who were post-menopausal, post-hysterectomy, and/or postoophorectomy (low Total $\mathrm{T}<5 \mathrm{ng} / \mathrm{dl}$ ) and pre-menopausal participants without a hysterectomy or oophorectomy (low Total $\mathrm{T}<15 \mathrm{ng} / \mathrm{dl}$ ). Average Total $\mathrm{T}$ for the pre-menopausal group was $23.55 \mathrm{ng} / \mathrm{dl} \quad(\mathrm{SD}=9.67) \quad$ (normal range:

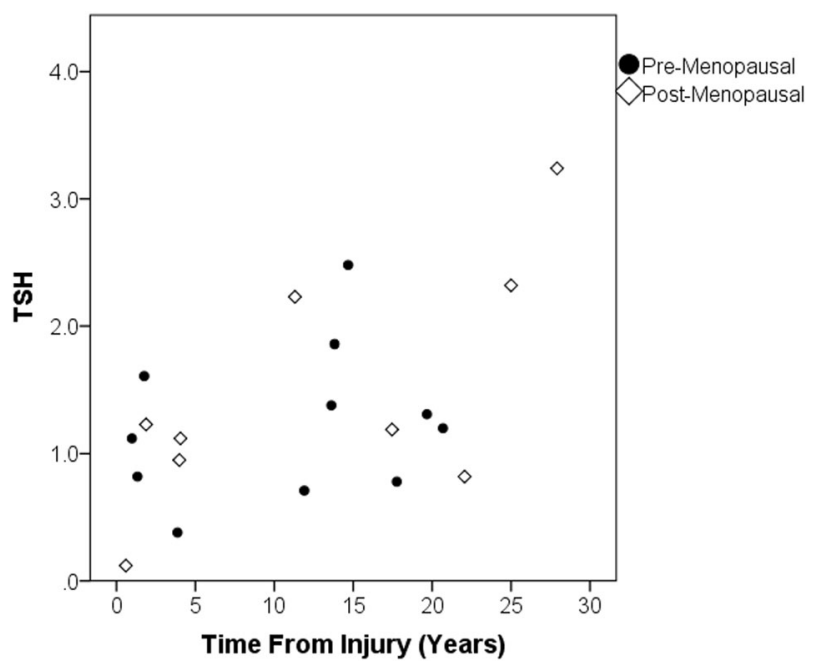

Fig. 1 Time from Injury and TSH Level. Scatter-plot represents the correlation between years from injury and TSH levels. Filled circles represent pre-menopausal women and diamonds represent postmenopausal women

$15-70 \mathrm{ng} / \mathrm{dl})$ and $15.89 \mathrm{ng} / \mathrm{dl}(\mathrm{SD}=11.1)$ (normal range: $5-51 \mathrm{ng} / \mathrm{dl}$ ) for the post-menopausal group. Low Total T was observed in four participants (one post-menopausal and post-hysterectomy; three pre-menopausal without hysterectomy/oophorectomy). Time from injury was significantly associated with TSH $(r=.536, p=.015$; Fig. 1$)$, such that greater time from injury was associated with elevated TSH levels. Total $\mathrm{T}$ was not significantly associated with time from injury $(r=-.180, p=.447)$. Age was not associated with TSH $(\rho=.213, p=.367)$ or Total T $(\rho=-.341$, $p=.141)$ in this sample.

\section{Center for Epidemiological Studies Depression Scale}

The average CES-D total score was 16.2 (SD 9.4). Four individuals fit the definition of "clinically irrelevant symptoms," seven had "subclinical depressive symptoms," and nine had "clinically relevant levels of depressive symptoms." Due to well-established changes across time in depression ratings, Pearson's correlation was used to assess the effect of time after SCI and age on CES-D total scores. No significant correlations were observed between age and CES-D total score $(r=-.047, p=.846)$. A significant association was found with time from injury and CES-D total score $(r=$ $-.547, p=.013$ ), such that lower CES-D total scores were associated with greater time from injury (Fig. 2).

\section{Association between hormone level and CES-D total score}

Due to the significant relationship between CES-D total score and time from injury in this sample, partial 


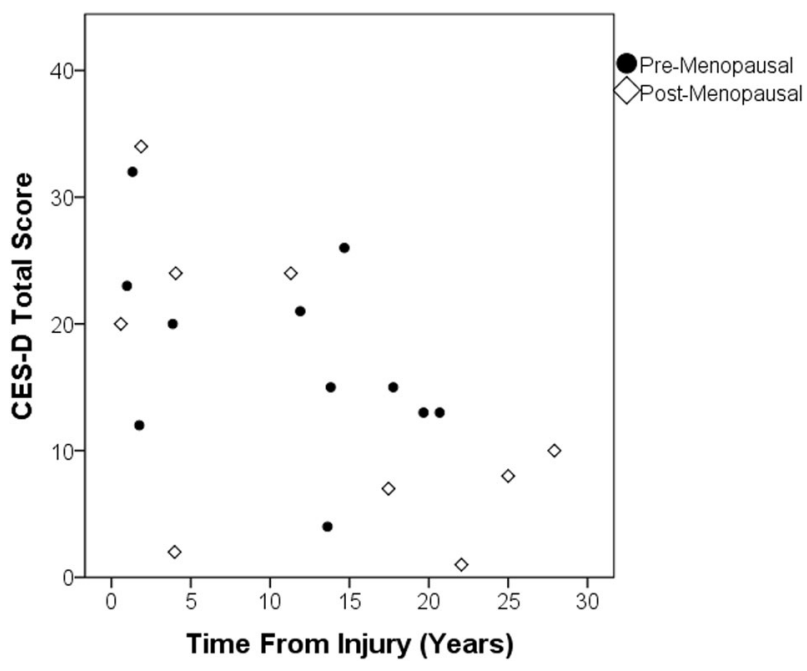

Fig. 2 Time from Injury and CES-D Total Score. Scatter-plot represents the correlation between years from injury and CES-D total score. Filled circles represent pre-menopausal women and diamonds represent post-menopausal women

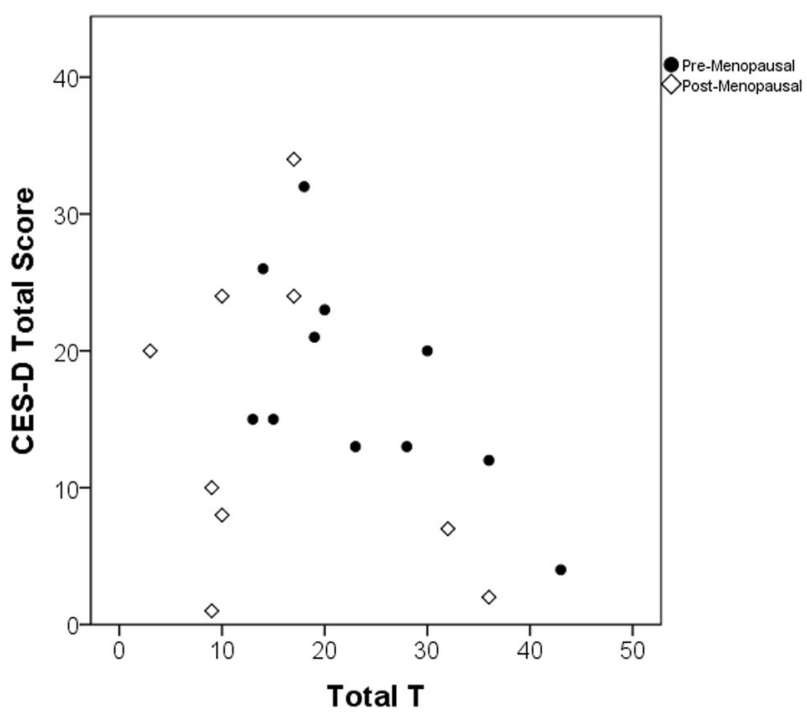

Fig. 3 Total T and CES-D Total Score. Scatter-plot represents the correlation between total testosterone (Total T) and Center for Epidemiological Studies Depression Scale (CES-D) total score. Raw scores, not residuals, are plotted. Filled circles represent premenopausal women and diamonds represent post-menopausal women

correlations were used to investigate the association between hormone levels and CES-D total score while controlling for time from injury. Partial correlations revealed that higher CES-D total scores were significantly associated with lower Total T levels $(\rho=-.536, p=.018$; Fig. 3); no significant associations were observed with TSH level $(\rho=.284, p=.239)$.
Table 3 Contingency table of individuals endorsing monthly sexual activity and clinically relevant depression (CES-D total >15)

\begin{tabular}{llll} 
& \multicolumn{2}{l}{$\begin{array}{l}\text { Clinically relevant } \\
\text { depression }\end{array}$} & Total \\
\cline { 2 - 3 } & Yes & No & \\
\hline Monthly sexual activity & 1 & 8 & 9 \\
Yes & 8 & 3 & 11 \\
No & 9 & 11 & 20 \\
Total & 9 & & \\
\hline
\end{tabular}

Fisher's exact test $p=.01$, Cramer's $V=.616$

\section{Association between monthly sexual activity and hormone levels, as well as depressive symptomology}

The Mann-Whitney $U$ tests revealed no significant effects of monthly sexual activity on hormone levels: Total T $(U=$ $27, p=.087)$ or TSH $(U=35, p=.270)$. The Fisher's exact test revealed a significant effect of monthly sexual activity for individuals with clinically relevant levels of depressive symptoms, such that the group that had monthly sexual activity had a lower proportion of individuals with clinically relevant levels of depressive symptoms $(p=.010$, Cramer's $V=.616$; Table 3).

\section{Discussion}

Approximately $80 \%$ of individuals with a SCI are male, producing research that has predominately focused on problems experienced by males [1]. This has direct consequences for women with SCI in that their unique concerns have not been adequately investigated. This study is one of the few studies investigating hormone levels in women after SCI. Results showed that four (20\%) participants presented with low total serum testosterone. Although only 20 participants were included in this study, the incidence of low testosterone was lower than estimates observed in males (43-46\%) [10-12].

In line with previous findings, a significant negative correlation was found between time from injury and CES-D ratings, representing decreased depressive symptoms in individuals with greater time from injury [34]. Furthermore, $45 \%$ of participants presented with "clinically relevant levels of depressive symptoms" and an additional 35\% of participants presented with "subclinical depressive symptoms." These results underscore the importance of depression screening and intervention in women with SCI, especially early phases post-injury.

Total serum testosterone was significantly correlated with symptoms of depression, such that low testosterone was associated with worse depressive symptomology. This finding 
is similar to previous research in both the able-bodied general [24] and able-bodied female [25] populations, such that abnormal testosterone levels (both low and high) were associated with depression. In this sample, Total $\mathrm{T}$ was not correlated with time from injury, which may provide evidence for a persistent change in total serum testosterone after SCI. TSH levels were significantly associated with time from injury, in that increased time from injury was associated with elevated TSH level. It should be noted that only one participant presented with a TSH level below the normal range and all other participants were within the normal range. Pre-injury and animal studies may be needed to clarify the development and effect of SCI on hormone levels in women after SCI.

Fifty-five percent of our sample engaged in monthly sexual activity. In this limited sample, there were no effects of monthly sexual activity on hormone levels (TSH and Total T), which have been previously observed [15-18]. Research exploring hormones and sexual dysfunction has found normal TSH levels in women with SCI [13], while studies in the general SCI population have identified high TSH levels [8]. There was a significant effect of sexual activity on depressive symptoms, such that individuals who had monthly sexual activity endorse clinically relevant levels of depressive symptoms (CES-D total $>15$ ) less than those who did not engage in monthly sexual activity. These results should be cautioned as psychological factors are only one modulator of sexual activity $[44,45]$. To provide a holistic approach to treatment for individuals with SCI, it has been suggested that pregnancy and sexual activity should become a part or greater part of SCI rehabilitation [44, 46-49].

One of the main barriers identified in this study was the blood draw timing requirement for women who were not postmenopausal; the blood draw timing protocol made it more difficult to complete the blood sampling at the time of the participants' enrollment as some participants were asked to return for the blood draw on another day. Although age, some medications, and neurological status are known to cause hormonal changes, this sample size was too small to investigate the effects of medication or neurological status on hormone levels or to detect age-related changes in hormone levels. Future studies should include an able-bodied control group and larger group sizes to better understand the specific SCI-related hormone changes and associations with depression in women.

The effects and consequences of SCI in women is underresearched. This study is one of a few studies specifically investigating thyroid function and testosterone levels and its relationship with depression in women with SCI. Results from this study showed a high rate of low testosterone (20\%) and depression (80\% were classified as having "subclinical" or "clinically relevant" depressive symptoms). In this sample low Total $\mathrm{T}$ was associated with depressive symptomology after accounting for time from injury. This study highlights the need for more research to elucidate the needs and concerns of women after SCI.

\section{Data archiving}

The datasets generated and/or analyzed during the current study are available from the corresponding author on reasonable request.

Funding This study was funded through a 2002 Sally Rynne National Association of Women's Health Quality Award.

\section{Compliance with ethical standards}

Conflict of interest The authors declare that they have no conflict of interest.

Ethical approval We certify that all applicable institutional and governmental regulations concerning the ethical use of human volunteers were followed during the course of this research.

Publisher's note: Springer Nature remains neutral with regard to jurisdictional claims in published maps and institutional affiliations.

\section{References}

1. National Spinal Cord Injury Statistical Center. Facts and figures at a glance [Internet]. Birmingham: University of Alabama at Birmingham; 2018. https://www.nscisc.uab.edu/Public/Facts\%20and \%20Figures\%20-\%202018.pdf.

2. Pinn VW. Women's health research: current state of the art. Glob Adv Health Med. 2013;2:8-10.

3. Pinn VW, Clayton JA, Begg L, Sass SE. Public partnerships for a vision for women's health research in 2020. J Women's Health 2002. 2010;19:1603-7.

4. Institute of Medicine. Women's health research: progress, pitfalls, and promise [Internet]. Washington, DC: The National Academies Press; 2010. https://www.nap.edu/catalog/12908/womens-healthresearch-progress-pitfalls-and-promise.

5. Sullivan SD, Nash MS, Tefera E, Tinsley E, Blackman MR, Groah S. Prevalence and etiology of hypogonadism in young men with chronic spinal cord injury: a cross-sectional analysis from two university-based rehabilitation centers. PMR. 2017;9:751-60.

6. Bauman WA, Spungen AM. Metabolic changes in persons after spinal cord injury. Phys Med Rehabil Clin N Am. 2000;11:109-40

7. Madduri SD, de Salvo E, Seebode JJ. Plasma androgens and estrogens in paraplegic men. Urology. 1979;13:179-81.

8. Prakash V, Lin MS, Song CH, Perkash I. Thyroid hypofunction in spinal cord injury patients. Paraplegia. 1980;18:56-63.

9. Wang YH, Huang TS, Lien IN. Hormone changes in men with spinal cord injuries. Am J Phys Med Rehabil. 1992;71:328-32.

10. Bauman WA, La Fountaine MF, Spungen AM. Age-related prevalence of low testosterone in men with spinal cord injury. $J$ Spinal Cord Med. 2014;37:32-9.

11. Durga A, Sepahpanah F, Regozzi M, Hastings J, Crane DA. Prevalence of testosterone deficiency after spinal cord injury. PMR. 2011;3:929-32. 
12. Barbonetti A, Vassallo MRC, Pacca F, Cavallo F, Costanzo M, Felzani G, et al. Correlates of low testosterone in men with chronic spinal cord injury. Andrology. 2014;2:721-8.

13. Lombardi G, Mondaini N, Macchiarella A, Popolo GD. Female sexual dysfunction and hormonal status in spinal cord injured (SCI) patients. J Androl. 2007;28:722-6.

14. Ueshiba H, Takeda S, Matoba H, Tanaka Y, Yuasa R, Tsuboi K, et al. Serum androgen levels in hyperthyroid women. Exp Clin Endocrinol Diabetes. 1997;105:359-62.

15. Jackson IM. The thyroid axis and depression. Thyroid J Am Thyroid Assoc. 1998;8:951-6.

16. Rack SK, Makela EH. Hypothyroidism and depression: a therapeutic challenge. Ann Pharm. 2000;34:1142-5.

17. Kuusi T, Taskinen MR, Nikkilä EA. Lipoproteins, lipolytic enzymes, and hormonal status in hypothyroid women at different levels of substitution. J Clin Endocrinol Metab. 1988;66:51-6.

18. Hutchinson KA. Androgens and sexuality. Am J Med. 1995;98 (1A):111S-5S.

19. Białek M, Zaremba P, Borowicz KK, Czuczwar SJ. Neuroprotective role of testosterone in the nervous system. Pol J Pharm. 2004;56:509-18.

20. Iqbal MJ, Dalton M, Sawers RS. Binding of testosterone and oestradiol to sex hormone binding globulin, human serum albumin and other plasma proteins: evidence for non-specific binding of oestradiol to sex hormone binding globulin. Clin Sci Lond Engl 1979. 1983;64:307-14.

21. Cheville AL, Kirshblum SC. Thyroid hormone changes in chronic spinal cord injury. J Spinal Cord Med. 1995;18:227-32.

22. Huang TS, Wang YH, Lai JS, Chang CC, Lien IN. The hypothalamus-pituitary-ovary and hypothalamus-pituitary-thyroid axes in spinal cord-injured women. Metabolism. 1996;45:718-22.

23. Pies RW. The diagnosis and treatment of subclinical hypothyroid states in depressed patients. Gen Hosp Psychiatry. 1997;19:344-54.

24. Joffe RT. Hormone treatment of depression. Dialogues Clin Neurosci. 2011;13:127-38.

25. Rohr UD. The impact of testosterone imbalance on depression and women's health. Maturitas. 2002;41 Suppl 1:S25-S46.

26. Goldstat R, Briganti E, Tran J, Wolfe R, Davis SR. Transdermal testosterone therapy improves well-being, mood, and sexual function in premenopausal women. Menopause. 2003;10:390-8.

27. Williams R, Murray A. Prevalence of depression after spinal cord injury: a meta-analysis. Arch Phys Med Rehabil. 2015;96:133-40.

28. Kessler RC, Berglund P, Demler O, Jin R, Koretz D, Merikangas $\mathrm{KR}$, et al. The epidemiology of major depressive disorder: results from the National Comorbidity Survey Replication (NCS-R). J Am Med Assoc. 2003;289:3095-105.

29. Krause JS, Kemp B, Coker J. Depression after spinal cord injury: relation to gender, ethnicity, aging, and socioeconomic indicators. Arch Phys Med Rehabil. 2000;81:1099-109.

30. Lim S-W, Shiue Y-L, Ho C-H, Yu S-C, Kao P-H, Wang J-J, et al. Anxiety and depression in patients with traumatic spinal cord injury: a nationwide population-based cohort study. PLoS ONE. 2017;12:e0169623.

31. Kraft R, Dorstyn D. Psychosocial correlates of depression following spinal injury: a systematic review. J Spinal Cord Med. 2015;38:571-83.
32. Williams RT, Wilson CS, Heinemann AW, Lazowski LE, Fann $\mathrm{JR}$, Bombardier $\mathrm{CH}$. Identifying depression severity risk factors in persons with traumatic spinal cord injury. Rehabil Psychol. 2014;59:50-6.

33. Gruener H, Zeilig G, Laufer Y, Blumen N, Defrin R. Increased psychological distress among individuals with spinal cord injury is associated with central neuropathic pain rather than the injury characteristics. Spinal Cord. 2018;56:176-84.

34. Saunders LL, Krause JS, Focht KL. A longitudinal study of depression in survivors of spinal cord injury. Spinal Cord. 2012;50:72-7.

35. Min J-A, Lee C-U, Hwang S-I, Shin J-I, Lee B-S, Han S-H, et al. The moderation of resilience on the negative effect of pain on depression and post-traumatic growth in individuals with spinal cord injury. Disabil Rehabil. 2014;36:1196-202.

36. Craig A, Tran Y, Guest R, Middleton J. Trajectories of selfefficacy and depressed mood and their relationship in the first 12 months following spinal cord injury. Arch Phys Med Rehabil. 2019;100:441-7.

37. Huang C-Y, Chen W-K, Lu C-Y, Tsai C-C, Lai H-L, Lin H-Y, et al. Mediating effects of social support and self-concept on depressive symptoms in adults with spinal cord injury. Spinal Cord. 2015;53:413-6.

38. Radloff LS. The CES-D Scale: a self-report depression scale for research in the general population. Appl Psychol Meas. 1977;1:385-401.

39. Wilson J, Foster D, Kronenberg H, Larsen P. Williams textbook of endocrinology. 9th ed. Philadelphia: W.B. Saunders Co; 1998.

40. Lewinsohn PM, Fenn DS, Stanton AK, Franklin J. Relation of age at onset to duration of episode in unipolar depression. Psychol Aging. 1986;1:63-8.

41. Radloff LS. 6/Use of the Center for Epidemiological StudiesDepression Scale with older adults. Clin Gerontol. 1986;5:119-36. PhD LT.

42. Roberts RE, Vernon SW. The Center for Epidemiologic Studies Depression Scale: its use in a community sample. Am J Psychiatry. 1983;140:41-6.

43. Weissman MM, Sholomskas D, Pottenger M, Prusoff BA, Locke BZ. Assessing depressive symptoms in five psychiatric populations: a validation study. Am J Epidemiol. 1977;106:203-14.

44. Kreuter M, Taft C, Siösteen A, Biering-Sørensen F. Women's sexual functioning and sex life after spinal cord injury. Spinal Cord. 2011;49:154-60.

45. Harrison J, Glass CA, Owens RG, Soni BM. Factors associated with sexual functioning in women following spinal cord injury. Spinal Cord. 1995;33:687-92.

46. Celik EC, Akman Y, Kose P, Arioglu P, Karatas M, Erhan B. Sexual problems of women with spinal cord injury in Turkey. Spinal Cord. 2014;52:313-15.

47. Sramkova T, Skrivanova K, Dolan I, Zamecnik L, Sramkova K, Kriz J, et al. Women's sex life after spinal cord injury. Sex Med. 2017;5:e255-9.

48. Tepper MS. Sexual education in spinal cord injury rehabilitation: current trends and recommendations. Sex Disabil. 1992;10:15-31.

49. Forsythe E, Horsewell JE. Sexual rehabilitation of women with a spinal cord injury. Spinal Cord. 2006;44:234-41. 